\title{
Acidentes de trabalho graves envolvendo membros superiores: Uma análise no contexto da crise brasileira
}

\author{
Serious work accidents involving upper limbs: An analysis in the context of the brazilian crisis \\ Accidentes de trabajo graves que involucran a miembros superiores: Un análisis en el contexto de
}

la crisis brasileña

Recebido: 15/05/2021 | Revisado: 19/05/2021 | Aceito: 20/05/2021 | Publicado: 07/06/2021

\author{
Alberto Pinto da Rosa Júnior \\ ORCID: https://orcid.org/0000-0003-0696-8090 \\ Universidade Federal de Uberlândia, Brasil \\ E-mail: albertojunior937@gmail.com \\ Thallys Rodrigues Félix \\ ORCID: https://orcid.org/0000-0003-3985-4428 \\ Universidade Federal de Uberlândia, Brasil \\ E-mail: thallysrodrigues@live.com \\ Luiz Renato Paranhos \\ ORCID: https://orcid.org/0000-0002-7599-0120 \\ Universidade Federal de Uberlândia, Brasil \\ E-mail: paranhos.lrp@gmail.com \\ Álex Moreira Herval \\ ORCID: https://orcid.org/0000-0001-6649-2616 \\ Universidade Federal de Uberlândia, Brasil \\ E-mail: alexmherval@ufu.br \\ Antônio Carlos Freire Sampaio \\ ORCID: https://orcid.org/0000-0003-1719-0245 \\ Universidade Federal de Uberlândia, Brasi \\ E-mail: antonio.sampaio@ufu.br
}

\section{Resumo}

Introdução: A crise econômica brasileira vivenciada na segunda década do século XXI exigiu transformações no mercado de trabalho, virtude do desemprego. Objetivo: Analisar os acidentes graves de trabalho ocorridos entre trabalhadores com e sem vínculo empregatício. Metodologia: Estudo observacional transversal de base documental e retrospectiva com dados da Ficha de Notificação de Acidente de Trabalho Grave, que informaram acidentes graves envolvendo membros superiores nos anos de 2016 a 2017. Os dados foram analisados por meio do Qui-quadrado de Pearson. Resultados: Foram analisadas 370 fichas de notificação compulsória. Observou-se uma alta prevalência dos acidentes graves entre homens da construção civil e marcenaria. Foi observada também uma maior prevalência de trabalhadores sem vínculo empregatício, os quais afirmaram ter menor dificuldade em operar o equipamento causador do acidente, mas participaram menos de atividades de treinamento e uso de equipamento de proteção individual. Conclusão: $O$ momento de crise econômica e, consequentemente no emprego, tem gerado um crescimento de trabalhadores sem vínculo, os quais são mais expostos aos acidentes graves de trabalho. Esse grupo de trabalhadores demandará mais atuação dos serviços públicos para desenvolver ações de treinamento e incentivo ao uso de proteção no trabalho.

Palavras-chave: Acidentes de trabalho; Saúde do trabalhador; Extremidade superior; Desemprego.

\begin{abstract}
Introduction: The Brazilian economic crisis experienced in the second decade of the 21 st century required changes in the labor market due to unemployment. Objective: To analyze the serious work accidents that occurred among workers with and without employment relationship. Methodology: Cross-sectional observational study with data from the Employee's Report of Injury Form, which reported major injuries involving upper limbs from 2016 to 2017. Data were analyzed using Pearson's chi-square. Results: 370 compulsory reporting forms were analyzed. There was a high prevalence of major injuries among construction and carpentry men. It was also observed a higher prevalence of workers without employment relationship, who said they had less difficulty in operating the equipment that caused the accident but participated less in training and use activities of personal protective equipment. Conclusion: The moment of economic crisis and, consequently in employment, has generated a growth of unrelated workers, who are more exposed to serious accidents at work. This group of workers will demand more action from public services to develop training and incentive actions to use protection at work.
\end{abstract}

Keywords: Occupational accidents; Occupational health; Upper extremity; Unemployment. 


\begin{abstract}
Resumen
Introducción: La crisis económica brasileña vivenciada en la segunda década del siglo XXI exigió transformaciones en el mercado de trabajo, en virtud del desempleo. Objetivo: Analizar los accidentes graves de trabajo ocurridos entre trabajadores con y sin vínculo laboral. Metodología: Estudio observacional transversal de base documental y retrospectiva con datos del Registro de Notificación de Accidente de Trabajo Grave, Brasil, que informaron accidentes graves que involucran los miembros superiores entre los años 2016 y 2017. Los datos fueron analizados por medio del Chi-cuadrado de Pearson. Resultados: Se analizaron 370 fichas de notificación obligatoria. Se observó una alta prevalencia de los accidentes graves entre hombres de la construcción civil y carpintería. Se observó también una mayor prevalencia de trabajadores sin vínculo laboral, los cuales afirmaron tener menos dificultar para operar el equipo causador del accidente, pero participaron menos en actividades de entrenamiento y de uso de equipos de protección individual. Conclusión: El momento de crisis económica y, consecuentemente de empleo, ha generado un crecimiento de trabajadores sin vínculo, los cuales quedan más expuestos a los accidentes graves de trabajo. Este grupo de trabajadores demandará más labor de los servicios públicos para desarrollar acciones de entrenamiento e incentivo al uso de protección en el trabajo.
\end{abstract}

Palabras clave: Accidentes de trabajo; Salud del trabajador; Extremidad superior; Desempleo.

\title{
1. Introdução
}

O Brasil vivenciou no início do século XXI um rápido crescimento da sua economia, contexto que foi alterado de forma pronunciada na segunda década do mesmo século. A crise econômica instalada no país se iniciou em função da crise global das commodities e se associou a um grande escândalo de corrupção, enfraquecendo a credibilidade e capacidade do governo nacional em enfrentar a crise (Melo, 2016). Dentre os efeitos sociais desta crise está o desemprego, cujas taxas cresceram de forma significativa ao longo dos anos de 2014 e 2015 (Castilhos, Fonseca \& Bavaresco, 2017; Melo, 2016).

$\mathrm{O}$ aumento do desemprego tem como consequências diretas as perdas financeiras, o endividamento das famílias e o aumento da violência (Vieira, Santos, Ocké-Reis \& Rodrigues, 2018). Para superar tal cenário, os trabalhadores assumem estratégias de enfrentamento e adaptação, produzindo novas formas de socialização, consumo e produção de renda (Castilhos et al., 2017). Nesse processo de reorganização social, as ocupações que envolvem trabalhos manuais e o próprio desemprego são responsáveis por impor um risco adicional à saúde do trabalhador (Glonti et al., 2015).

Como medida de enfrentamento da crise, o governo brasileiro assumiu medidas de austeridade como parte de uma agenda neoliberal, trazendo impactos em diversas áreas sociais (Doniec, Dall'Alba, \& King, 2016). É importante compreender que essas medidas também impõem risco a saúde do trabalhador e estão associadas a aumento dos acidentes de trabalho (Costa, Costa \& Cintra, 2018). Esses acidentes ocorrem principalmente nos membros superiores, chegando a atingir 45,5\% do total de acidentes de trabalho graves notificados (Cordeiro et al., 2005; Santana et al., 2009; Scussiato, Sarquis, Kirchhof \& Kalinke, 2013).

Considerando o cenário de crise e o desemprego vivenciados no Brasil, bem como as consequentes modificações nas relações trabalhistas, este estudo tem por objetivo analisar os acidentes graves de trabalho ocorridos entre trabalhadores com e sem vínculo empregatício.

\section{Metodologia}

\section{Desenho do estudo e Critérios Éticos}

Trata-se de um estudo observacional transversal de base documental e retrospectiva com dados secundários de acesso restrito. A ferramenta Reporting of Observational Studies in Epidemiology (STROBE) foi utilizada para nortear o desenvolvimento da pesquisa e apresentação dos resultados dessa pesquisa (Von Elm et al., 2014). O estudo foi desenvolvido de acordo com os princípios da Declaração de Helsinki e com a Resolução nº466 de 2012 do Conselho Nacional de Saúde. Dessa forma, o projeto foi analisado e aprovado previamente a sua execução pelo Comitê de Ética em Pesquisa com Seres Humanos da Universidade Federal de Uberlândia. 


\section{Contexto de Estudo}

O estudo foi desenvolvido a partir dos dados armazenados por uma unidade do Centro de Referência em Saúde do Trabalhador (CEREST), referência para 28 municípios do interior de Minas Gerais. Foram considerados os acidentes de trabalho graves envolvendo membros superiores.

\section{Fontes de Dados e Amostragem}

Os dados foram coletados da Ficha de Notificação de Acidente de Trabalho Grave, componente do Sistema de Informação de Agravos de Notificação (SINAN). Do montante de fichas notificadas foram incluídos no estudo aqueles que informaram acidentes graves envolvendo membros superiores (dedos, mãos, punho, braço, antebraço e ombro) notificados nos anos de 2016 a 2017.

\section{Variáveis em Estudo}

Foram coletados dados sociodemográficos e ocupacionais, por meio das variáveis numéricas (idade) e categóricas (sexo, estado civil, escolaridade, situação no mercado, abertura de Comunicação de Acidentes de Trabalho (CAT), participação em treinamento, utilização de Equipamento de Proteção Individual (EPI), dificuldade de executar a tarefa e tipo de atividade desenvolvida pelo trabalhador. A variável dependente desse estudo foi a situação no mercado, dicotomizada em trabalhadores formais e informais.

\section{Análise Estatística}

Os dados foram tabulados e analisados utilizando o Software SPSS ${ }^{\circledR}$ (versão 21.0). A variável "situação no mercado" foi considerada como desfecho do presente estudo, organizada de forma dicotômica (com vínculo e sem vínculo empregatício). O tipo de atividade desenvolvida pelo trabalhador apresentou-se de forma muito dispersa, e optou-se por reagrupá-los de acordo com a Classificação Brasileira de Ocupações (Classificação Brasileira de Ocupações [CBO], 2010).

Inicialmente foi realizada exploração e a análise descritiva das variáveis em estudo, considerando as medidas de frequência. A correlação entre as variáveis independentes (abertura de CAT, realização de treinamento, utilização de EPI, dificuldade em desempenhar a atividade e ocorrência do acidente durante a manutenção do equipamento) e a variável desfecho (vínculo empregatício) foi testada por meio do Qui-quadrado de Pearson, considerando o nível de significância menor que 5\%. Foi verificada ainda a Razão de Prevalência do grupo com vínculo empregatício em relação ao grupo sem vínculo empregatício.

\section{Resultados}

Foram analisados 370 Fichas de Notificação de Acidentes de Trabalho Graves relacionadas à acidente de trabalho graves em membros superiores no período de 2016 a 2017, nos quais foi observada uma maior prevalência desse tipo de acidente entre os trabalhadores sem vínculo empregatício $(n=215 ; 58,1 \%)$. A idade desses trabalhadores variou de 13 a 82 anos $(40,76 \pm 13,81)$ anos. As características sociodemográficas dos trabalhadores vítimas de acidentes de trabalhos em membros superiores e a classificação da sua ocupação segundo a CBO (2010) estão apresentadas na Tabela 1. 
Tabela 1: Caracterização sociodemográfica dos trabalhadores vítimas de acidente de trabalho graves em membros superiores, Uberlândia (MG), 2019.

\begin{tabular}{|c|c|c|c|c|c|c|}
\hline & \multicolumn{2}{|c|}{ Total } & \multicolumn{2}{|c|}{ Com vínculo } & \multicolumn{2}{|c|}{ Sem vínculo } \\
\hline & $\mathrm{N}$ & $\%$ & $\mathrm{~N}$ & $\%$ & $\mathrm{~N}$ & $\%$ \\
\hline \multicolumn{7}{|l|}{ Sexo } \\
\hline Masculino & 345 & 93,2 & 154 & 41,6 & 191 & 51,6 \\
\hline Feminino & 25 & 6,8 & 18 & 4,9 & 7 & 1,9 \\
\hline \multicolumn{7}{|l|}{ Estado Civil } \\
\hline Casado & 201 & 54,3 & 83 & 22,4 & 118 & 31,9 \\
\hline Solteiro & 106 & 28,7 & 56 & 15,1 & 50 & 13,5 \\
\hline Divorciado & 3 & 0,8 & 1 & 0,3 & 2 & 0,5 \\
\hline Viúvo & 3 & 0,8 & 0 & 0,0 & 3 & 0,8 \\
\hline União estável & 3 & 0,8 & 2 & 0,5 & 1 & 0,3 \\
\hline Ignorado & 54 & 14,6 & 30 & 8,1 & 24 & 6,5 \\
\hline \multicolumn{7}{|l|}{ Nível de escolaridade } \\
\hline Analfabeto & 5 & 1,4 & 2 & 0,5 & 3 & 0,8 \\
\hline $1^{\circ}$ Grau incompleto & 84 & 22,7 & 30 & 8,1 & 54 & 14,6 \\
\hline $1^{\circ} \mathrm{Grau}$ completo & 48 & 13,0 & 21 & 5,7 & 27 & 7,3 \\
\hline $2^{\circ}$ Grau incompleto & 37 & 10,0 & 23 & 6,2 & 14 & 3,8 \\
\hline $2^{\circ} \mathrm{Grau}$ completo & 42 & 11,4 & 25 & 6,7 & 17 & 4,6 \\
\hline Superior incompleto & 5 & 1,4 & 4 & 1,1 & 1 & 0,3 \\
\hline Superior completo & 17 & 4,6 & 9 & 4,2 & 8 & 2,2 \\
\hline Ignorado & 132 & 35,5 & 58 & 15,7 & 74 & 20,0 \\
\hline \multicolumn{7}{|c|}{ Código Brasileiro de Ocupações } \\
\hline Grupo 0 & 2 & 0,5 & 2 & 0,5 & 0 & 0,0 \\
\hline Grupo 1 & 5 & 1,4 & 4 & 1,1 & 1 & 0,3 \\
\hline Grupo 2 & 20 & 5,4 & 16 & 4,3 & 4 & 1,1 \\
\hline Grupo 3 & 33 & 8,9 & 22 & 5,9 & 11 & 0,3 \\
\hline Grupo 4 & 0 & 0,0 & 0 & 0,0 & 0 & 0,0 \\
\hline Grupo 5 & 34 & 9,2 & 18 & 4,9 & 16 & 4,3 \\
\hline Grupo 6 & 29 & 7,8 & 12 & 3,2 & 17 & 4,6 \\
\hline Grupo 7 & 169 & 45,7 & 47 & 12,7 & 122 & 33,0 \\
\hline Grupo 8 & 18 & 4,9 & 17 & 4,6 & 1 & 0,3 \\
\hline Grupo 9 & 43 & 11,6 & 22 & 5,9 & 21 & 5,7 \\
\hline Ignorado & 17 & 4,6 & 12 & 3,2 & 5 & 1,4 \\
\hline
\end{tabular}

Grupo 0: Membros das forças armadas, policiais e bombeiros; Grupo 1: Membros superiores do poder público, dirigentes de organizações de interesse público e de empresas, gerentes; Grupo 2: Profissionais das ciências e das artes; Grupo 3: Técnicos de nível médio; Grupo 4: Trabalhadores de serviços administrativos; Grupo 5: Trabalhadores dos serviços, vendedores do comércio em lojas e mercados; Grupo 6: Trabalhadores agropecuários, florestais e da pesca; Grupo 7: Trabalhadores da produção de bens e serviços industriais; Grupo 8: Trabalhadores da produção de bens e serviços industriais; Grupo 9: Trabalhadores em serviços de reparação e manutenção. Fonte: Autores.

Na Tabela 1 é possível observar a caracterização sociodemográfica, evidenciando uma maior prevalência desses acidentes entre os trabalhadores do sexo masculino $(n=345 ; 93,2 \%)$ e com estado civil casado (221; 54,3\%). Quanto ao nível de escolaridade, observou-se que os dados se mostraram dispersos, com predomínio do primeiro grau incompleto, além de um elevado valor de informação ignorada (35,7\%). A organização dos trabalhadores segundo a CBO evidenciou uma concentração no Grande Grupo 7 - Trabalhadores da Produção de Bens e Serviços Industriais (n = 169, 45,7\%), sobressaindo nesse montante os trabalhadores da construção civil $(n=65 ; 17,6 \%)$ e os marceneiros $(n=31 ; 8.4 \%)$.

A distribuição dos acidentes de trabalho de acordo com a parte do corpo atingida e o tipo de lesão, e a sua classificação em relação a presença ou não de vínculo empregatício é apresentada na Tabela 2. 
Tabela 2: Caracterização das lesões graves ocorridas em membros superiores, Uberlândia (MG), 2019.

\begin{tabular}{lcccccc}
\hline & \multicolumn{2}{c}{ Total } & \multicolumn{2}{c}{ Com vínculo } & \multicolumn{2}{c}{ Sem vínculo } \\
\cline { 2 - 7 } & $\mathrm{N}$ & $\%$ & $\mathrm{~N}$ & $\%$ & $\mathrm{~N}$ & $\%$ \\
\hline Parte do corpo atingida & & & & & & \\
Dedo & 162 & 43,8 & 71 & 43,8 & 91 & 56,2 \\
Mão & 81 & 21,9 & 37 & 45,7 & 44 & 54,3 \\
Braço & 86 & 23,2 & 40 & 46,5 & 46 & 53,5 \\
Punho & 17 & 4,6 & 11 & 64,7 & 6 & 35,3 \\
Cotovelo & 4 & 1,1 & 1 & 25,0 & 3 & 75,0 \\
Ombro & 6 & 1,6 & 3 & 50,0 & 3 & 50,0 \\
Antebraço & 5 & 1,4 & 5 & 100,0 & 0 & 0,0 \\
Não especificado & 5 & 1,4 & 3 & 60,0 & 2 & 40,0 \\
Tendão & 4 & 1,1 & 1 & 25,0 & 3 & 75,0 \\
\hline Tipo de Lesão & & & & & & \\
Fratura & 207 & 55,9 & 95 & 45,9 & 112 & 54,1 \\
Corte & 63 & 17,0 & 31 & 49,2 & 32 & 50,8 \\
Amputação & 49 & 13,2 & 25 & 51,0 & 24 & 49,0 \\
Esmagamento & 13 & 3,5 & 8 & 61,5 & 5 & 38,5 \\
Queimadura & 10 & 2,7 & 4 & 40,0 & 6 & 60,0 \\
Lesão ou trauma & 9 & 2,4 & 3 & 33,3 & 6 & 66,7 \\
Outros & 15 & 4,2 & 6 & 40,0 & 9 & 60,0 \\
Ignorado & 4 & 1,1 & 0 & 0,0 & 4 & 100,0 \\
\hline
\end{tabular}

Fonte: Autores.

Observa-se uma importante prevalência de acidentes graves de trabalho envolvendo dedos ( $\mathrm{n}=162 ; 43,8 \%)$, seguido por mão $(n=81 ; 21,9 \%)$ e braço $(n=86 ; 23,2 \%)$, e a menor prevalência corresponde ao tendão $(n=4 ; 1,1 \%)$. Quanto ao tipo de lesão prevaleceram aquelas caracterizadas como fratura $(n=207 ; 55,9 \%)$, seguida por corte ( $n=63 ; 17 \%)$ e amputação $(\mathrm{n}=49 ; 13,2 \%)$.

A análise e correlação das medidas de proteção entre os grupos de trabalhados com vínculo e sem vínculo empregatício está disposta na Tabela 3.

Tabela 3: Análise da prevalência de medidas de proteção ao trabalho entre trabalhadores com vínculo e sem vínculo empregatício. Uberlândia (MG), 2016-2017.

\begin{tabular}{|c|c|c|c|c|c|c|}
\hline & \multicolumn{2}{|c|}{ Com vínculo } & \multicolumn{2}{|c|}{ Sem vínculo } & \multirow{2}{*}{$\begin{array}{c}\text { Valor de } \\
\text { p }\end{array}$} & \multirow{2}{*}{$\begin{array}{c}\text { Razão de Prevalência } \\
\text { (Com vínculo/Sem vínculo) }\end{array}$} \\
\hline & $\mathbf{N}$ & $\%$ & $\mathbf{N}$ & $\%$ & & \\
\hline \multicolumn{7}{|c|}{ Abertura de Comunicação de Acidente de Trabalho $(n=240)$} \\
\hline Sim & 76 & 31,7 & 9 & 3,8 & \multirow{2}{*}{$>0,001 *$} & \multirow{2}{*}{$6,581(3,517-12,312)$} \\
\hline Não & 47 & 17,5 & 108 & 47,1 & & \\
\hline \multicolumn{7}{|c|}{ Participação em treinamento $(n=335)$} \\
\hline Sim & 46 & 13,7 & 32 & 9,6 & \multirow{2}{*}{$>0,001 *$} & \multirow{2}{*}{$1,546(1,166-2,049)$} \\
\hline Não & 94 & 28,1 & 163 & 48,7 & & \\
\hline \multicolumn{7}{|c|}{ Uso de Equipamento de Proteção Individual (n=283) } \\
\hline Sim & 51 & 18,0 & 43 & 15,2 & \multirow{2}{*}{0,008} & \multirow{2}{*}{$1,365(1,067-1,746)$} \\
\hline Não & 71 & 25,1 & 118 & 41,7 & & \\
\hline \multicolumn{7}{|c|}{ Relato de dificuldade em realizar a tarefa $(n=334)$} \\
\hline Sim & 21 & 6,3 & 38 & 11,4 & \multirow{2}{*}{0,256} & \multirow{2}{*}{$0,875(0,705-1,086)$} \\
\hline Não & 120 & 35,9 & 155 & 46,4 & & \\
\hline \multicolumn{7}{|c|}{ Ocorrência do acidente durante a manutenção de equipamento $(n=357)$} \\
\hline Sim & 30 & 8,4 & 35 & 9,8 & \multirow{2}{*}{0,553} & \multirow{2}{*}{$1,075(0,841-1,374)$} \\
\hline Não & 123 & 34,5 & 169 & 47,3 & & \\
\hline
\end{tabular}

Fonte: Autores. 
Foi verificada uma relação estatisticamente significante para a abertura de CAT, participação em treinamentos e a utilização de EPI. A razão de prevalência mostrou que a chance de os trabalhadores registrados abrirem a CAT é 6,58 vezes maior que sem vínculo. Seguindo a mesma lógica de análise, a chance de os trabalhadores com vínculo participarem de treinamentos é 1,54 vezes maior; e a chance de os trabalhadores com vínculo utilizarem EPI é 1,36 vezes maior. Os trabalhadores sem vínculo afirmaram ter menor dificuldade em operar o equipamento causador do acidente, mas essa correlação não se apresentou como estatisticamente significativa.

\section{Discussão}

Ao avaliar os acidentes graves de trabalho ocorridos no período de 2016 e 2017, observou-se que esses acidentes ocorrem em maior prevalência entre os trabalhadores sem vínculo formal. Dentre os membros superiores estudados, prevaleceram as lesões em dedo e do tipo fratura. Os trabalhadores informais também apresentam um menor uso de medidas de proteção contra acidentes de trabalho. Esse resultado confirma a hipótese de que os trabalhadores sem vínculo empregatício estão mais expostos a ocorrência de acidentes graves de trabalho, uma vez que utilizam em menor frequência as medidas protetivas que poderiam reduzir os acidentes de trabalho.

A parte do corpo atingida pela lesão grave foi semelhante a estudo de outros autores (Cordeiro et al., 2005; Molina et al., 2016; Zangirolani, Cordeiro, Medeiros \& Stephan, 2008). Para Molina et al. (2016), que também realizaram estudos a partir de um CEREST, mas não se restringiram quando aos membros superiores, as partes mais atingidas foram punho e da mão. Para Cordeiro et al. (2005), cuja investigação partiu de um sistema de vigilância que abrange serviços hospitalares e de pronto-atendimento, as mãos representaram 32,3\% dentre as partes do corpo atingidas. No estudo de Zangirolani et al. (2008) as partes do corpo mais atingidas foram as mãos e os pés. Cabe destacar que esses estudos não segmentaram dedos e mãos, como o presente estudo. Essa diferenciação é importante uma vez que no presente estudo os acidentes apenas com dedos somaram o dobro daqueles que afetam mão.

Um dado demográfico importante de ser destacado é a maior ocorrência dos acidentes graves de trabalho entre os trabalhadores do sexo masculino, superando percentualmente todos os estudos sobre acidentes de trabalho realizados no Brasil: Fonseca, Mazzer, Barbieri e Elui (2006) observaram a predominância do sexo masculino em 74,4\%; Souza, Cabral, Sampaio e Mancini (2008) observaram que esse dado atingiu 87\%; no estudo de de Oliveira, Sime, Costa e Ferrigno (2013) esse dado chegou a 92\%; Rossi, Ferrigno e da Cruz (2011) observaram que os acidentes com membros superiores de uma unidade hospitalar alcançaram 59\% da amostra. Estes dados divergem da tendência de redução dessa discrepância entre os sexos que vinha sendo demonstrada (Gavioli, de Oliveira \& Mathias, 2017; Souza et al., 2008). Uma possibilidade para o expressivo predomínio do sexo masculino pode estar relacionada às ocupações mais observadas pelo estudo (construção civil e marcenaria), nas quais se observa maior inserção de homens.

É notório o efeito da crise sobre a mudança no perfil de vínculo empregatício (Castilhos et al., 2017). Em estudos prévios, sobre acidentes de trabalho prevaleciam os vínculos formais: de Oliveira et al. (2013) observaram que o vínculo formal atingia 89,3\%; Molina et al. (2016) aferiram que entre os anos analisados (2009, 2010 e 2011) os vínculos celetistas variaram entre $79,0 \%$ e $90,6 \%$. No presente estudo, os trabalhadores com vínculo empregatício corresponderam a apenas 41,9\%. Este é um fator preocupante e que deve objetivo de vigilância em saúde do trabalhador, uma vez que as condições de trabalho dos trabalhadores sem vínculo empregatício são perigosas e associadas à diversos fatores de risco para a saúde, além da ausência de medidas de proteção ao trabalhador (Rios, Nery, Rios, Casotti \& Cardoso, 2015). É importante também observar, uma vez que a legislação atual ampliou a terceirização, que terceirizadas tendem a investir menos em medidas de proteção (Costa et al., 2018). 
O fato da maior ocorrência de abertura CAT entre os trabalhadores com vínculo empregatício não gerou surpresa aos pesquisadores. Esse dado já era esperado, uma vez que a abertura de CAT é obrigatória apenas para trabalhadores públicos e privados regidos pela Consolidação das Leis do Trabalho. Apesar de compreensível que as notificações sejam obrigadas apenas à essa parte dos trabalhadores, a exclusão dos trabalhadores sem vínculo forma pode estar agravando o quadro de subnotificação de acidentes de trabalho já existentes (Cordeiro et al., 2005; Molina et al., 2016).

Apesar dos trabalhadores sem vínculo empregatício alegarem não ter dificuldade em realizar a ação causadora do acidente grave de trabalho, a participação em ações de treinamento para executar o trabalho e o uso de equipamentos de proteção foram menores nesse grupo.

Como tem sido comum aos estudos com dados secundários oriundos de fichas de notificação, a presença de subnotificações ou mesmo de notificações incompletas é um fator preocupante. Neste estudo, para algumas variáveis analisadas, o número de dados ignorados foi expressivo e pode ter afetado a caracterização dos acidentes de trabalho graves envolvendo membros superiores. Nesse sentido, Tipple, Souza, Almeida, Sousa e Siqueira (2004) e de Oliveira et al. (2013) chamam a atenção para que os registros de acidentes de trabalho precisam de investimentos, para se conter as subnotificações e aumentar a conscientização do trabalhador e dos órgãos públicos, no tocante à prevenção e reabilitação, evitando a incapacidade do trabalhador e prejuízos para as empresas e instituições públicas.

\section{Conclusão}

Os resultados desta investigação chamam a atenção para a insuficiência do uso da CAT, e o que ela pode acarretar aos órgãos de vigilância em saúde do trabalhador e para a elaboração de estratégias de enfrentamento aos acidentes de trabalho, uma vez que essa ferramenta não é obrigatória para trabalhadores sem vínculo empregatício. Dessa forma, os dados epidemiológicos a partir desse instrumento são incompletos e ignoram uma parcela crescente dos trabalhadores em tempo de crise, aqueles sem vínculo empregatício.

Ainda diante do contexto de aumento do volume de trabalhadores sem vínculo empregatício, os resultados deste estudo indicam a necessidade de os órgãos públicos desenvolverem ações de treinamento e estímulo ao uso de equipamentos de proteção. Estas funções classicamente estavam sob a responsabilidade das empresas, contudo, observando o crescimento acelerado de trabalhadores sem vínculo, é essencial que o poder público se torna mais ativo nesse campo, em especial os CEREST, visando um cuidado integral ao trabalhador.

\section{Referências}

Castilhos, R. B., Fonseca, M. J., \& Bavaresco, V. (2017). Consumption, crisis, and coping strategies of lower class families in Brazil: A sociological account. International Journal of Consumer Studies, 41(4), 379-388.

Classificação Brasileira de Ocupações (2010). ( $3^{\mathrm{a}}$ ed). Brasília: MTE/SPPE.

Cordeiro, R., Vilela, R. A. G., Medeiros, M. A. T. D., Gonçalves, C. G. D. O., Bragantini, C. A., Varolla, A. J., \& Celso, S. (2005). O sistema de vigilância de acidentes do trabalho de Piracicaba, São Paulo, Brasil. Cadernos de Saúde Pública, 21(5), 1574-1583.

Costa, B. S., Costa, S. D. S., \& Cintra, C. L. D. (2018). Os possíveis impactos da reforma da legislação trabalhista na saúde do trabalhador. Rev Bras Med Trab, 16(1), 109-117.

Doniec, K., Dall'Alba, R., \& King, L. (2016). Austerity threatens universal health coverage in Brazil. The Lancet, 388(10047), 867-868.

Estratégia Nacional para Redução dos Acidentes do Trabalho 2015-2016 (2015). Brasília, DF: Ministério do Trabalho e do Emprego.

Fonseca, M. D. C. R., Mazzer, N., Barbieri, C. H., \& Elui, V. M. C. (2006). Traumas da mão: estudo retrospectivo. Rev bras ortop, 41(5), 181-6.

Gavioli, A., de Oliveira, M. L. F., \& Mathias, T. A. D. F. (2017). Potencialidade de notificação e informação de acidentes de trabalho no acolhimento com classificação de risco. Revista Uningá, 52(1). 
Glonti, K., Gordeev, V. S., Goryakin, Y., Reeves, A., Stuckler, D., McKee, M., \& Roberts, B. (2015). A systematic review on health resilience to economic crises. PloS one, 10(4), e0123117.

Melo, M. A. (2016). Latin America's new turbulence: crisis and integrity in Brazil. Journal of Democracy, 27(2), 50-65.

Molina, A. C., de Lima, M. A. F., Fressatti, W. L., Neto, C. A. P., Junior, A. L. C., \& Lima, S. A. M. (2016). Notificações de Acidentes de Trabalho em CEREST de Botucatu-SP. Revista Laborativa, 5(1), 64-79.

Oliveira, T. P., Sime, M. M., Costa, J. F. B., \& Ferrigno, I. S. V. (2013). Estudo retrospectivo dos acidentes traumáticos da mão relacionados ao trabalho/Retrospective study of work related traumatic hand injuries. Cadernos Brasileiros de Terapia Ocupacional, 21(2).

Rios, M. A., Nery, A. A., Rios, P. A. A., Casotti, C. A., \& Cardoso, J. P. (2015). Fatores associados a acidentes de trabalho envolvendo trabalhadores informais do comércio. Cadernos de Saúde Pública, 31, 1199-1212.

Rossi, T. N., Ferrigno, I. S. V., \& da Cruz, D. M. C. (2011). Prevalência de acidentes de trabalho com lesão do membro superior em uma universidade do interior do estado de São Paulo. Cadernos brasileiros de terapia ocupacional, 19(2).

Santana, V. S., Xavier, C., Moura, M. C. P., Oliveira, R., Espírito-Santo, J. S., \& Araújo, G. (2009). Gravidade dos acidentes de trabalho atendidos em serviços de emergência. Revista de Saúde Pública, 43, 750-760.

Scussiato, L. A., Sarquis, L. M. M., Kirchhof, A. L. C., \& Kalinke, L. P. (2013). Perfil epidemiológico dos acidentes de trabalho graves no Estado do Paraná, Brasil, 2007 a 2010. Epidemiologia e Serviços de Saúde, 22(4), 621-630.

Souza, M. A. P., Cabral, L. H. D. A., Sampaio, R. F., \& Mancini, M. C. (2008). Acidentes de trabalho envolvendo mãos: casos atendidos em um serviço de reabilitação. Fisioterapia e Pesquisa, 15(1), 64-71.

Tipple, A. F. V., Souza, A. C. S., Almeida, A. N. G. D., Sousa, S. B. D., \& Siqueira, K. M. (2004). Acidente com material biológico entre trabalhadores da área de expurgo em centros de material e esterilização.

Vieira, F. S., Santos, I. S., Ocké-Reis, C., \& Rodrigues, P. H. A. (2018). Políticas sociais e austeridade fiscal: como as políticas sociais são afetadas pelo austericídio da agenda neoliberal no Brasil e no mundo.

Von Elm, E., Altman, D. G., Egger, M., Pocock, S. J., Gøtzsche, P. C., Vandenbroucke, J. P., \& Strobe Initiative. (2014). The Strengthening the Reporting of Observational Studies in Epidemiology (STROBE) Statement: guidelines for reporting observational studies. International journal of surgery, 12(12), 14951499 .

Zangirolani, L. T. O., Cordeiro, R., Medeiros, M. A. T. D., \& Stephan, C. (2008). Topologia do risco de acidentes do trabalho em Piracicaba, SP. Revista de Saúde Pública, 42, 287-293. 